\title{
How Late Can You Update Gaze-contingent Multi- resolutional Displays Without Detection?
}

\author{
LESTER C. LOSCHKY \\ Kansas State University \\ and \\ GARY S. WOLVERTON \\ University of Illinois at Urbana-Champaign
}

\begin{abstract}
This study investigated perceptual disruptions in gaze-contingent multi-resolutional displays (GCMRDs) due to delays in updating the center of highest resolution after an eye movement. GCMRDs can be used to save processing resources and transmission bandwidth in many types of single-user display applications such as virtual reality, video-telephony, simulators, and remote piloting. The current study found that image update delays as late as $60 \mathrm{~ms}$ after an eye movement did not significantly increase the detectability of image blur and/or motion transients due to the update. This is good news for designers of GCMRDs, since $60 \mathrm{~ms}$ is ample time to update many GCMRDs after an eye movement without disrupting perception. The study also found that longer eye movements led to greater blur and/or transient detection, due to moving the eyes further into the lowresolution periphery, effectively reducing the image resolution at fixation prior to the update. In GCMRD applications where longer saccades are more likely (e.g., displays with relatively large distances between objects), this problem could be overcome by increasing the size of the region of highest resolution.
\end{abstract}

Categories and Subject Descriptors: H.5.2 [Information Interfaces and Presentation]: User Interfaces - Usercentered Design, Windowing Systems, Screen Design, Evaluation/Methodology; I.3.3 [Picture/Image Generation]: Viewing algorithms; I.4.2 [Image Processing and Computer Vision]: Compression (Coding); General Terms: Design, Human Factors, Experimentation.

Additional Key Words and Phrases: gaze-contingent, level of detail, multiresolution, foveation, foveated, area of interest, display updates, perceptual compression, eyetracking, eye movements, saccades, saccadic suppression, visual perception, contrast thresholds, blur detection, peripheral vision, bandwidth

\section{INTRODUCTION}

Users of virtual reality, simulations, video-telephony, teleoperation, and other single-user applications often need large, high-resolution displays exceeding limits on bandwidth and/or computation resources. One way around these limitations is to eliminate detail that users cannot resolve in the visual periphery. Gaze-contingent multi-resolutional

This paper is a major revision of a paper presented at the HFES Annual Meeting 2005, Sept. 2005, Orlando, FL. The paper presented there appears in the Proceedings of the Human Factors and Ergonomics Society 49th Annual Meeting - 2005. This research was supported by funds from the Beckman Institute, the University of Illinois Research Board, the U.S. Army Research Laboratory under the Federated Laboratory Program, Cooperative Agreement DAAL01-96-2-0003, and the Eastman Kodak Company. Eyetracking equipment was purchased with funds from the National Institute of Mental Health, and other essential equipment was provided by the National Science Foundation under Grant CDA 96-24396.

Authors' addresses: Lester C. Loschky, Department of Psychology, Kansas State University, Manhattan, KS, 66506; e-mail: loschky@ksu.edu; Gary Wolverton, Beckman Institute, University of Illinois at UrbanaChampaign, 61801; e-mail: wolverton@uiuc.edu.

Permission to make digital/hard copy of part of this work for personal or classroom use is granted without fee provided that the copies are not made or distributed for profit or commercial advantage, the copyright notice, the title of the publication, and its date of appear, and notice is given that copying is by permission of the ACM, Inc. To copy otherwise, to republish, to post on servers, or to redistribute to lists, requires prior specific permission and/or a fee.

(C) 2006 ACM 1073-0516/01/0300-0034 \$5.00 
displays (GCMRDs) do just that, by dynamically displaying high resolution information wherever the user is looking, as indicated by a gaze-tracker, and lower resolution elsewhere [for reviews, see: Duchowski and Coltekin 2007, Duchowski, et al. 2004, Geisler and Perry 1999, Loschky and McConkie 2000, Parkhurst and Niebur 2002, Reingold, et al. 2003]. Human factors research on GCMRDs has primarily focused on two key questions: (1) What are the limits of peripheral visual resolution when viewing scene images [Geri and Zeevi 1995, Loschky, et al. 2005, Luebke, et al. 2000, Sere, et al. 2000, Yang, et al. 2001]? and (2) What are the perception and performance costs associated with reducing image resolution below those limits [Kortum and Geisler 1996, Loschky and McConkie 2002, Loschky and McConkie 2000, Parkhurst, et al. 2000, Reingold and Loschky 2002, Shioiri and Ikeda 1989, Watson, et al. 1997]? However, far fewer studies have tried to answer another critical question for designers of GCMRDs: How late can you update the center of highest resolution after the user has moved their eyes, without disrupting perception or performance? The current study provides an answer to this question.

The top panel of Figure 1 schematically represents the image update process that must occur at the end of each saccade. In A, an eye indicates the user's gaze position on the screen, the white circle around it represents the region of highest resolution, the surrounding gray area represents the lower-resolution over the rest of the image, and the arrow indicates an eye movement into that area. B shows the eye at the new gaze location, before the update. After some delay, the high resolution area is updated to the new gaze location, as shown in C. The question investigated here concerns the effects of delays in this updating process. While ideally one would update the display immediately at the end of each saccade (i.e., eye movement), this is impossible in practice because it takes time to identify when a saccade has ended and where the eyes are, to render the new multi-resolutional image, to transmit it, and to display it. Rendering the image, alone, can take between 25-150 ms [Geisler and Perry 1999, Ohshima, et al. 1996, Thomas and Geltmacher 1993].

Such updating delays could cause perceptual difficulties in two ways. First, when a new fixation begins, prior to the update the fixated region still has reduced image resolution, which may hinder perception. Second, when the update occurs, the change in image resolution may be perceived as a motion transient, which may disrupt perception 

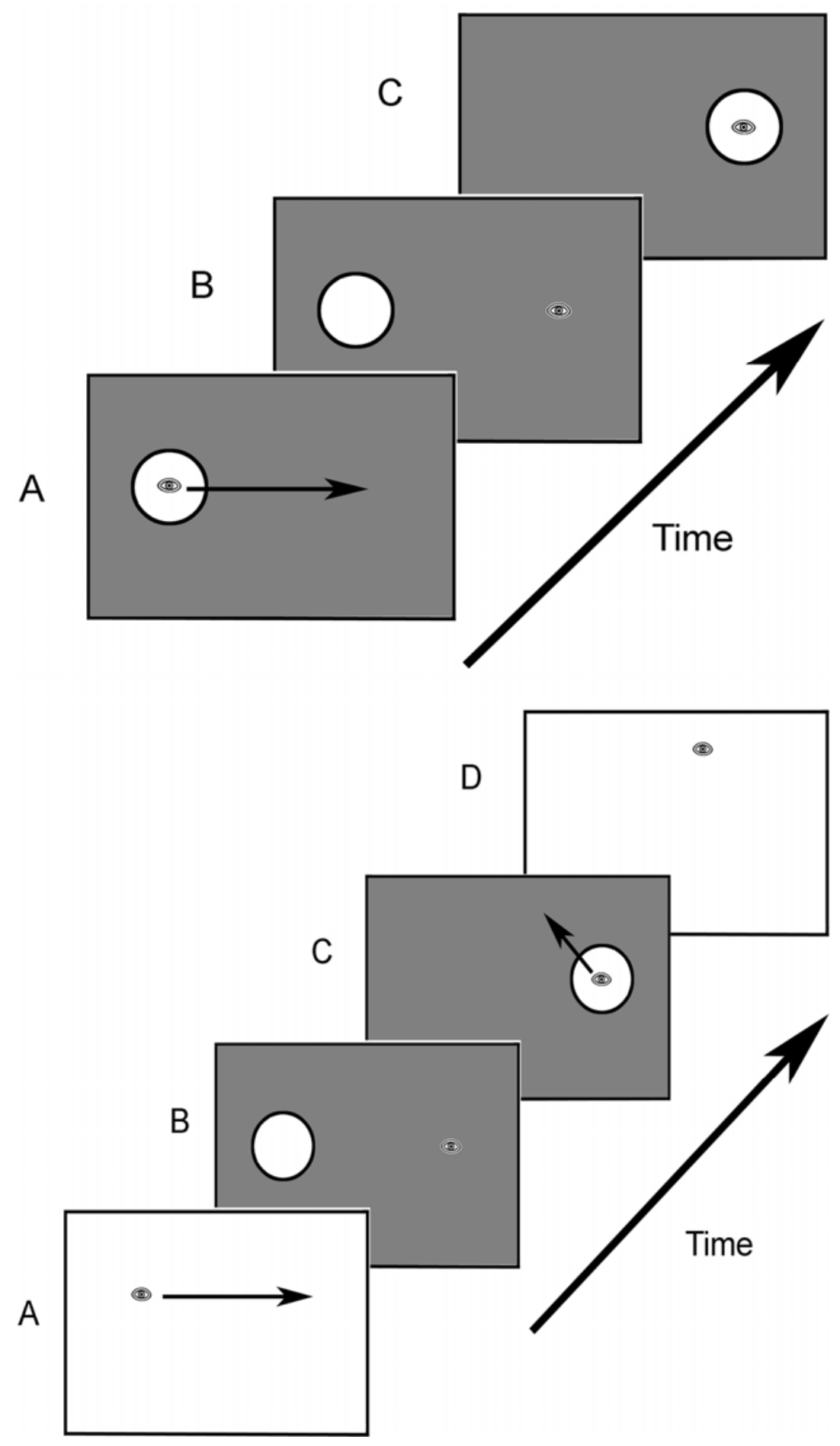

Figure 1. Top: Schematic of a GCMRD over time. Bottom: Same for a dual delayed occasional GCMRD. 
[Reingold and Stampe 2000]. However, saccadic suppression raises perceptual thresholds for low spatial frequencies and motion signals just before, during, and for $\sim 20$ $80 \mathrm{~ms}$ after each saccade [Burr, et al. 1994, October 6, Ross, et al. 2001, Shioiri 1993, Volkmann, et al. 1978], which may help reduce sensitivity to image blur and motion transients.

Simulator studies have shown that image update delays in excess of the apparent limits of post-saccadic suppression impair both perception and task performance [Grunwald and Kohn 1994, Turner 1984] and can cause simulator sickness [c.f., Draper, et al. 2001, Frank, et al. 1988]. Turner and colleagues varied delays between 130 to 280 $\mathrm{ms}$, and found that path following and target identification performance decreased as update delays increased. Given such extreme update delays, the Turner, et al. results are unsurprising. However, a more recent study of the detection of post-saccadic image resolution changes indicated that delays greater than only $5 \mathrm{~ms}$ after a saccade are detectable, with asymptotic detection reached at delays as short as $30 \mathrm{~ms}$ post-saccade [McConkie and Loschky 2002]. While these results might suggest that only extremely short update delays can go undetected, it is unclear how relevant that study is to answering the key question here, because it did not involve a GCMRD; instead the study made switches from normal images to completely low-pass filtered images, and then back again. Thus, studies are needed that use GCMRDs and measure the perception and performance effects of update delays within the post-saccadic suppression period. One such study [Loschky and McConkie 2000, Experiment 6], used a GCMRD with update delays of 5, 15 and $45 \mathrm{~ms}$, and found that a $45 \mathrm{~ms}$ delay did not affect search performance but did increase fixation durations. Such effects of update delays on fixation durations may reflect increased processing difficulty, or they might reflect saccadic inhibition [Reingold and Stampe 2000], that is the inhibition of the impulse to move the eyes, which has been shown to occur in response to visual transients during a fixation, such as those produced by update delays in gaze-contingent displays [Hodgson, et al. 1993, van Diepen and Wampers 1998].

\section{THE CURRENT STUDY}

Based on the previous results reviewed above, it is difficult to say how late one can update a GCMRD without disrupting perception. Our own previous results [Loschky and McConkie 2000, Experiment 6] suggest that GCMRD update delays should be less than $45 \mathrm{~ms}$ to avoid disrupting perceptual processes, as reflected by increased fixation durations. However, if the effects of update delays on fixation durations are caused by 
saccadic inhibition, it would make the applied consequences less clear. In contrast, the detection task is generally considered to be the simplest, most sensitive psychophysical measure of conscious perception. From an applied perspective, if the update delays of a GCMRD are undetectable, the system has been maximized for perceptual quality on that dimension. That said, it is technically non-trivial to measure update delay detection. Indeed, the current study is, to our knowledge, the first ever to have done so.

Detection of GCMRD update delays should interact with detection of image degradation (obviously, updating a normal image with an identical copy of itself should be imperceptible regardless of the update delay - it is the presence of detectable image blur at the new gaze position, or the change in image resolution caused by the update, that makes the update delay potentially detectable). The current study carefully controls for the image degradation factor by using established GCMRD blur detection thresholds, with image filtering levels derived from a model of eccentricity-dependent contrast sensitivity [Loschky, et al. 2005]. By using a range of image filtering levels that bracket the blur detection threshold, it is possible for the first time to determine whether update delays can push otherwise undetectable image blur above the detection threshold.

In order to study GCMRD update delay detection thresholds, one must use a display system optimized to minimize update delays, so that at least some delays are below threshold. The current study uses a display system proven capable of producing large display changes that are undetectable because they occur so quickly after a saccade [McConkie and Loschky 2002]. Furthermore, in order to bracket the update delay detection threshold, the current study uses a wide range of delays $(5-80 \mathrm{~ms})$ spanning the known post-saccadic suppression period.

In sum, the current study directly measures image update delay effects on conscious perception of image degradation, by having viewers detect image blur or motion transients in a GCMRD, while factorially varying delay and image filtering levels. The study uses a relatively wide range of update delays and a range of image filtering levels that bracket the blur detection threshold, in order to determine the relationship between update-delay and blur-detection thresholds.

\subsection{Method}

2.2.1 Participants. Twelve paid undergraduate and graduate students participated. All had $20 / 30$ or better uncorrected vision. 
2.2.2 Stimuli. Stimuli were 24 monochrome photographic scene images $\left(18^{\circ} \times 12^{\circ}\right.$, $768 \times 512$ pixels), with varied subject matter (from street scenes, to building interiors) and much visual detail (see example in Figure 2). In order to achieve the goal of producing a large corpus of eye movement data, only scenes meeting the subjective criterion of having numerous, different, small- to medium-sized objects were chosen, because such images would require subjects to make many eye fixations in order to encode all their details.

Images were filtered using an algorithm developed at Eastman Kodak Company, described in detail elsewhere [Loschky, et al. 2005], which is a modified version of Geisler and Perry's [1998] foveated multi-resolution pyramid. The current study used three levels of image filtering to bracket the updating detection threshold. We varied filtering using a parameter corresponding to E2 for grating resolution (the retinal eccentricity at which visual resolution drops to half maximum). The filtering E2 values were $6.22^{\circ}, 3.11^{\circ}$, and $1.55^{\circ}$ (Figure 2). Previous research [Loschky, et al. 2005] using a $5 \mathrm{~ms}$ update initiation deadline, found that image filtering at the predicted blur detection threshold, E2 $=3.11^{\circ}$, was almost never detected, while one octave greater filtering, E2 = $1.55^{\circ}$, was often detected (60\%), and filtering one octave below the predicted threshold, $\mathrm{E} 2=6.22^{\circ}$, was never detected. Filtering condition was a within-subjects' variable that was counterbalanced across images and participants.

2.2.3 Apparatus. As in several of our previous GCMRD studies [Loschky and McConkie 2002, Loschky, et al. 2005, McConkie and Loschky 2002], the current study used an eye-tracker with high spatial accuracy (Dual Purkinje Image Generation 5), and a system designed to minimize image-update delays. Eye position was sampled at 1000 $\mathrm{Hz}$, providing high temporal resolution for identifying the ends of saccades. We avoided on-line image generation time by pre-computing 330 versions of each image (in a $22 \times 15$ imaginary grid over the image) and storing them in a 2 GB randomly accessible image memory and display controller. Thus, wherever viewers' eyes were directed on critical fixations when a modified image was present, there was an image version whose center of high-resolution was within $0.41^{\circ}$ of gaze position. The $60 \mathrm{~Hz}$ monitor could be updated at any point during the refresh cycle, so updates were completed within $17 \mathrm{~ms}$ of initiation. 


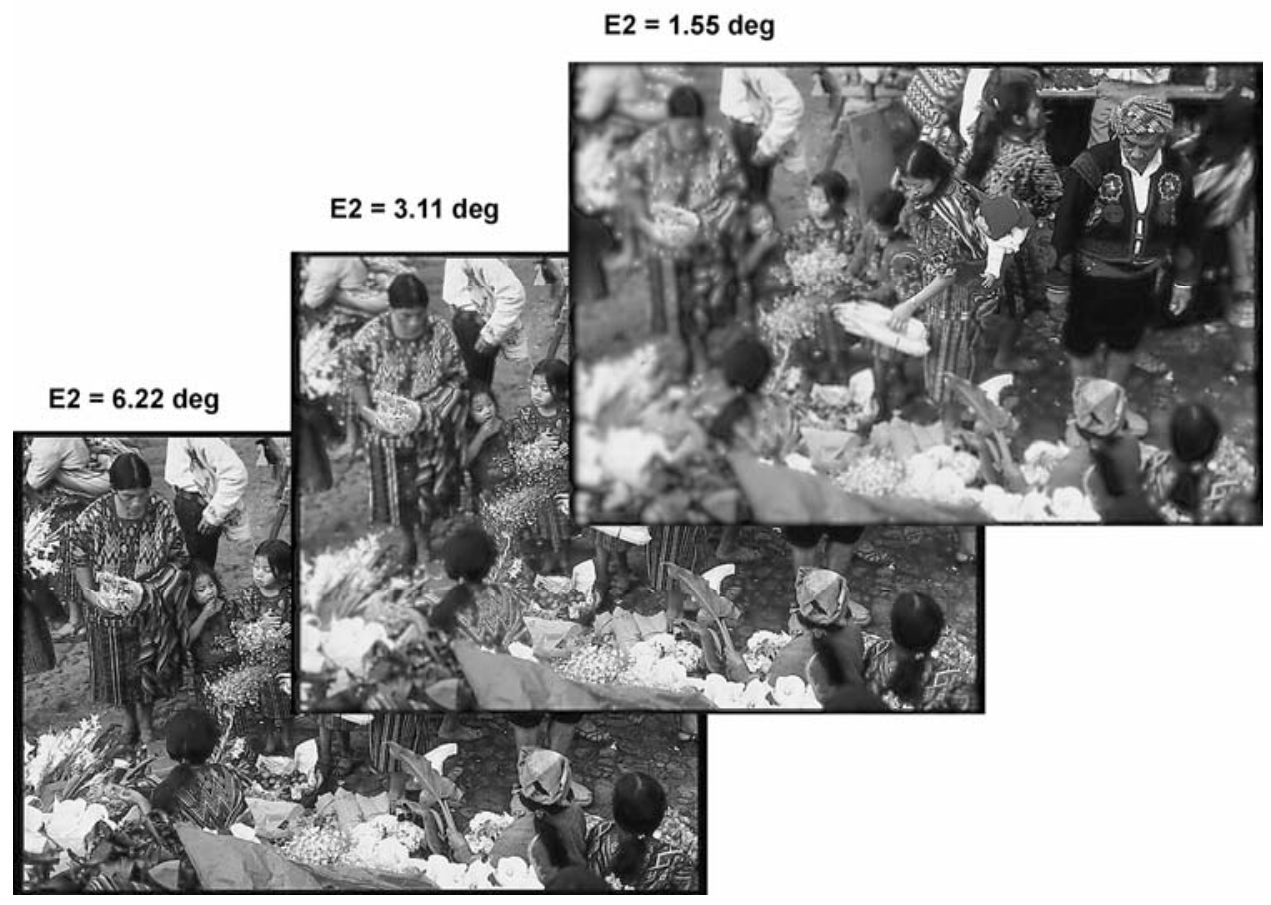

Figure 2. A set of 3 example images for filtering levels E2 $=6.22,3.11$, and 1.55 degrees.

2.2.4 Procedures. In order to examine conscious perception of the visual artifacts produced by update delays in a GCMRD, we combined elements of the delayed window paradigm used in our previous study of the effects of image update delays [Loschky and McConkie 2000, Experiment 6] and the occasional window detection paradigm used in Loschky, et al. [2005], which we call the "dual delayed occasional window" paradigm. The current paradigm is shown schematically in the bottom panel of Figure 1. On most fixations, viewers saw an all high-resolution image, represented schematically by white space (Figure 1, bottom panel, A). However, at the onset of occasional critical saccades, ranging from the $9^{\text {th }}$ to the $11^{\text {th }}$, the high-resolution image was replaced with a multiresolutional image, whose center of highest resolution was put at the preceding gaze location (fixation n-1). Thus, when the eyes landed at the new gaze location, they would likely be in a lower-resolution region (B), as in a normal GCMRD before updating of the area of interest (c.f., top panel, B). Then, after a delay of 5, 20, 40, 60, or $80 \mathrm{~ms}$, the center of highest-resolution was put at the new center of gaze (bottom panel, C; c.f., top panel, C). Finally, as soon as the next saccade was initiated, the constant high-resolution image was returned (D). Thus, the viewer's task was to detect the blur or update-related 
motion transient (panels B and C) that occurred for only a single critical fixation. This pattern continued for the duration of each 30 second trial. (Subjects were not told about these contingencies.) As a control condition to measure subjects' false alarm rates, we included "no-change" display changes in which the high-resolution image was replaced by an identical copy of itself after the same update delays and filtering conditions as the real changes. Image update delay was a within-subjects variable, with the particular update delay for each critical fixation chosen randomly.

To motivate participants to carefully look at the images, thus producing many eye movements, and therefore many potentially detectable display changes, we asked them to do several tasks. There were 24 trials each in four different tasks $(4 \times 24=96$ total trials per subject), with each of the 24 scene images shown a total of four times, once in each task for each subject: "short-term learning" (scrutinize the pictures in preparation for a test after seeing a subset of 6 scene images), "short-term test" (scrutinize the pictures and then decide if they are the same or different from the subset you just studied), "long-term learning" (scrutinize each of the entire set of 24 pictures again, after which you will be tested on your memory for all of them), and "blur/transient detection only" (scrutinize each of the 24 images to see if you can detect occasional blur or motion transients, as an indirect measure of memory). Our chief interest was only in subjects' blur and/or transient detection.

\subsection{Results}

Our primary analyses examined the effects of image filtering levels and image updating delays on the detection of image blur and/or motion transients in GCMRDs. Further analyses examined the effects of saccade length on detection rates. In addition, a preliminary analysis examined whether task affected detection rates.

2.3.1 Precursors. Before carrying out our main analyses, we first cleaned the data by excluding blinks, extreme outlying reaction times (top and bottom 1\%), extremely short fixation durations and saccade lengths (bottom 1\%), and errors in positioning the center of highest resolution $\geq 2^{\circ}$ on either fixation $n$ or fixation $n-1$. After all exclusions ( $15 \%$ of the original total), 4,494 critical fixations remained, and in the majority of cases the center of highest resolution was quite accurately placed (placement error for fixation $\mathrm{n}-1$ : $\mathrm{M}=0.32^{\circ}, \operatorname{Mdn}=0.33^{\circ}$, Mode $=0.38^{\circ}$; placement error for fixation $\mathrm{n}: \mathrm{M}=0.52^{\circ}, \mathrm{Mdn}=$ $0.47^{\circ}$, Mode $=0.42^{\circ}$. 
2.3.2 Perceptibility of Image Degradation as a Function of Task. Each of the filtering levels occurred equally often in each task $\left(\chi^{2}(6, \mathrm{~N}=3152)=1.47, \mathrm{p}=.96\right.$, n.s. $)$. An analysis of detection rates as a function of task, excluding subject 5 who was missing data in one task, showed that task had a neglibible effect $(\mathrm{F}(3,30)=2.02, \mathrm{p}=.133$, n.s. $)$. Therefore, in all further analyses, detection data were combined across tasks.

2.3.3 Perceptibility of Image Degradation as a Function of Filtering Level. As expected based on the results of Loschky, et al. [2005], we found a significant effect of filtering level on detection, $F(1.66,18.26$, Geisser-Greenhouse adjusted $)=138.21, p<$ .001 . As shown in Figure 3, the false alarm rate was essentially zero, and subjects almost never detected filtering level E2 $=6.22^{\circ}$, rarely detected filtering level E2 $=3.11^{\circ}$, but frequently detected filtering level $\mathrm{E} 2=1.55^{\circ}$.

2.3.4 Perceptibility of Image Degradation as a Function of Delay. Viewers never detected a change from the first multi-resolutional image to an all high-resolution image after a delay of $5 \mathrm{~ms}$, replicating McConkie and Loschky [2002] in a GCMRD, and confirming that the $5 \mathrm{~ms}$ delay can serve as a no-delay baseline. Figure 3 shows that update delays significantly increased detection, $\mathrm{F}(4,44)=5.68, \mathrm{p}=.001$, and interacted with filtering level, $\mathrm{F}(12,132)=1.83, \mathrm{p}=.048$. This interaction is because updates have no effect when image blur is completely undetectable (filtering level E2 $=6.22$ ). Nevertheless, while blur in level E2 $=3.11$ was very rarely detected, an $80 \mathrm{~ms}$ update delay significantly increased detection relative to the $5 \mathrm{~ms}$ baseline $(\mathrm{F}(1,11)=6.67, \mathrm{p}=$ $.025)$, though no other delays did so. The same was true for the more detectable filtering level E2 $=1.55$ (80 ms vs. $5 \mathrm{~ms}, \mathrm{~F}(1,11)=4.94, \mathrm{p}=.048$, all other comparisons n.s.).

2.3.5 Perceptibility of Image Degradation as a Function of Saccade Length. A further analysis investigated the effect of saccade length on detection of blur. In subdividing the data by saccade length for this further analysis, the number of observations in each cell was reduced, resulting in empty cells for some subjects. Thus, only 8 subjects' data was included in the analyses. Saccades were divided into three equal-frequency trintiles: saccades $<1.43^{\circ}, 1.43^{\circ} \geq$ saccades $\leq 3.32^{\circ}$, and saccades $>3.32^{\circ}$. As shown in Figure 4, longer saccades led to higher detection rates $(\mathrm{F}(2,14)=24.79, \mathrm{p}<.001)$, though only in the detectable filtering conditions $(\mathrm{F}(1.59,11.16$, Geisser-Greenhouse adjusted $)=4.17, \mathrm{p}$ $=.052$ ). The increased detectability of image blur following longer saccades did not differ as a function of update delay $(\mathrm{F}(8,56)=1.35, \mathrm{p}=.240$, n.s. $)$, nor was there a 3 way interaction between saccade length, filtering level and delay $(\mathrm{F}(16,112)=1.12, \mathrm{p}=$ .345 , n.s.). These non-effects are likely due to the small numbers of observations in each 


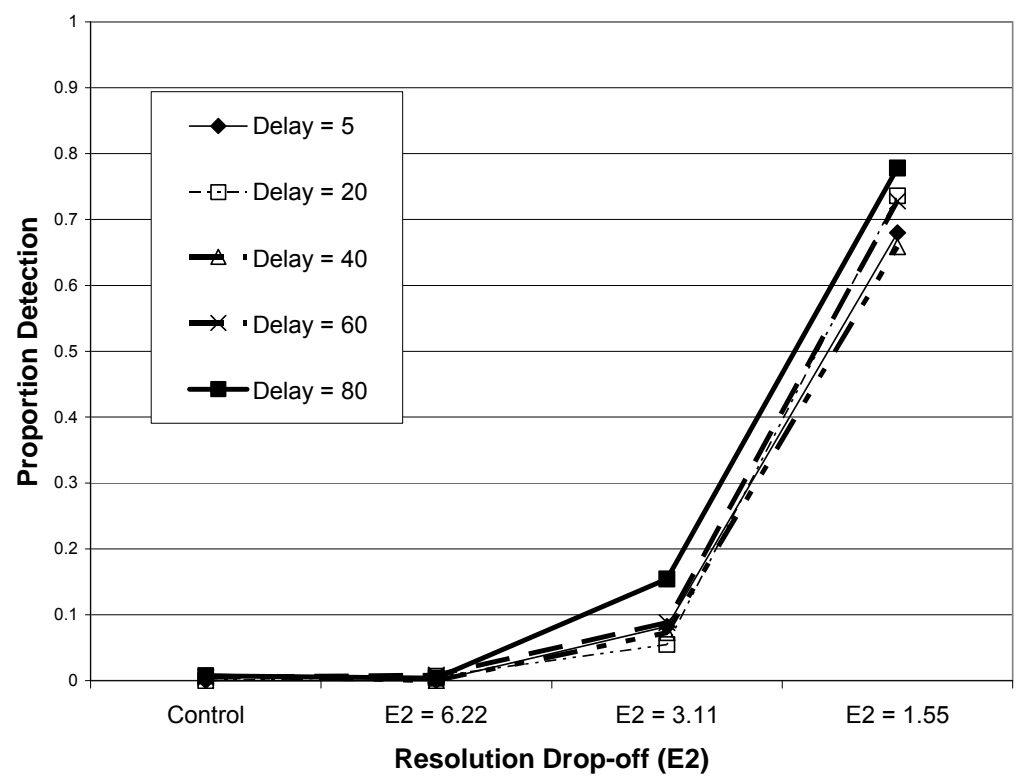

Figure 3. Proportion detection of occasionally presented gaze-contingent multiresolutional images as a function of filtering level (control, E2 $=6.22,3.11$, and 1.55 degrees) and update delay $(5,20,40,60$, and $80 \mathrm{~ms})$.

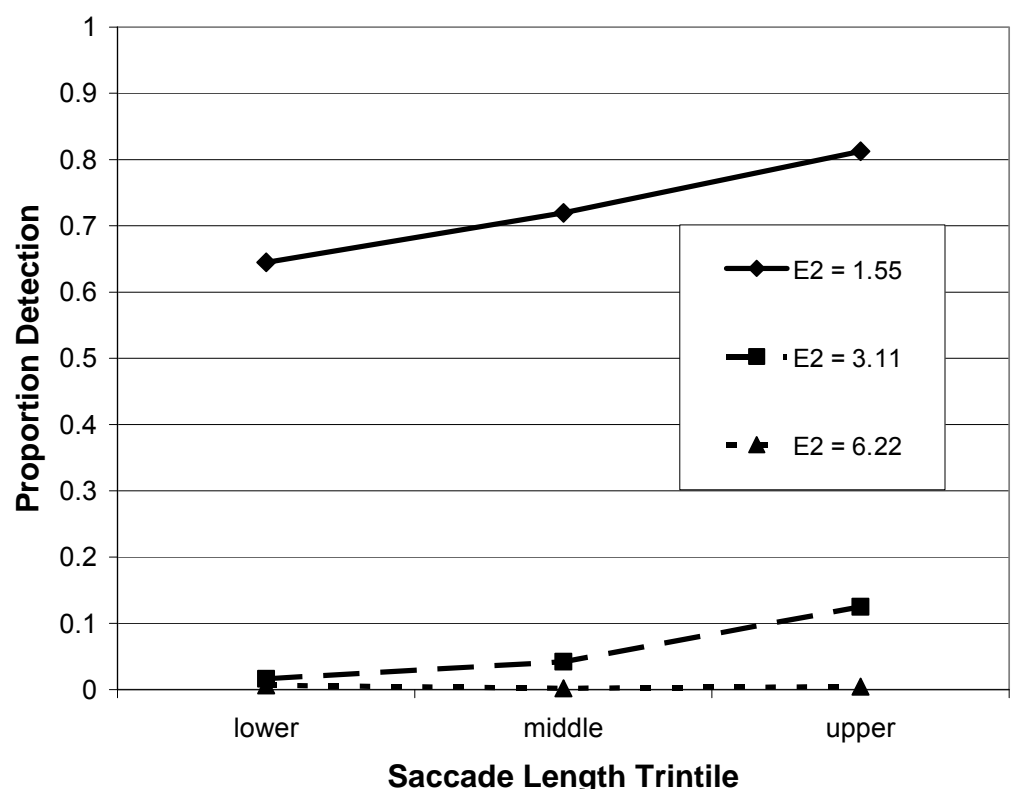

Figure 4. Proportion detection of occasionally presented gaze-contingent multi-resolutional images as a function of filtering level (control, E2 $=6.22,3.11$, and 1.55 degrees) and preceding saccade length trintile (lower, middle, upper). Saccade lengths in degrees for the three trintiles were: lower trintile $<1.43^{\circ}, 1.43^{\circ} \geq$ middle trintile $\leq 3.32^{\circ}$, and upper trintile $>3.32^{\circ}$. 
cell. Nevertheless, there was a strong visual trend in the data suggesting that, in the E2 = 3.11 filtering level, in which blur was very rarely detected, the strongest effect of delay was found following the longest saccades.

2.3.5 Perceptibility of Image Degradation as a Function of Saccade Length. A further analysis investigated the effect of saccade length on detection of blur. In subdividing the data by saccade length for this further analysis, the number of observations in each cell was reduced, resulting in empty cells for some subjects. Thus, only 8 subjects' data was included in the analyses. Saccades were divided into three equal-frequency trintiles: saccades $<1.43^{\circ}, 1.43^{\circ} \geq$ saccades $\leq 3.32^{\circ}$, and saccades $>3.32^{\circ}$. As shown in Figure 4, longer saccades led to higher detection rates $(\mathrm{F}(2,14)=24.79, \mathrm{p}<.001)$, though only in the detectable filtering conditions $(\mathrm{F}(1.59,11.16$, Geisser-Greenhouse adjusted $)=4.17, \mathrm{p}$ $=.052$ ). The increased detectability of image blur following longer saccades did not differ as a function of update delay $(\mathrm{F}(8,56)=1.35, \mathrm{p}=.240$, n.s. $)$, nor was there a 3 way interaction between saccade length, filtering level and delay $(F(16,112)=1.12, p=$ .345 , n.s.). These non-effects are likely due to the small numbers of observations in each cell. Nevertheless, there was a strong visual trend in the data suggesting that, in the E2 = 3.11 filtering level, in which blur was very rarely detected, the strongest effect of delay was found following the longest saccades.

\section{DISCUSSION}

The current study has provided, to our knowledge, the first test of the effect of update delays on the detectability of image blur and/or motion transients in GCMRDs. Update delays had a relatively small but significant impact on conscious perception of image degradation. As predicted, long update delays $(80 \mathrm{~ms})$ significantly increased detection of image blur that was otherwise almost never detected. However, for update delays $\leq 80$ $\mathrm{ms}$, and filtering levels bracketing the blur detection threshold, the effect of update delay was much less than that of filtering level, and, as would be expected, for filtering well below the blur detection threshold, delays had no impact. Interestingly, the current study did not find significantly increased detection relative to the $5 \mathrm{~ms}$ delay baseline for update delays $\leq 60 \mathrm{~ms}$, though our previous research found increased fixation durations for $45 \mathrm{~ms}$ delays [Loschky and McConkie 2000, Experiment 6]. This difference may have been due to using more detectable image blur in the previous study, or to differences between factors affecting eye movements and conscious detection processes. Of particular interest to designers of GCMRDs is the fact that delays as long as $60 \mathrm{~ms}$ did 
not significantly increase blur detection, which is good news because it provides ample time for many GCMRDs [e.g., Geisler and Perry 1999, Ohshima, et al. 1996] to update their displays without causing visible artifacts.

The analysis of the effects of saccade length indicated that following longer eye movements, image blur was more detectable. Longer saccades take the eyes farther from the center of highest resolution located at the preceding gaze location (fixation $\mathrm{n}-1$ ), thus the eyes will land in a region of lower image resolution. Thus, longer saccades effectively reduce the image resolution available at fixation, resulting in greater blur detection. The results also showed that as the update delay increased, image blur becomes increasingly noticeable. It would therefore seem to follow that the strongest effects of delay should be found after the longest saccades. (Conversely, a tiny saccade, of say $0.2^{\circ}$, would not even necessarily trigger a change in the location of the point of highest resolution, thus eliminating the update altogether.) However, the data did not show such an interaction. In fact, the data somewhat supported this explanation, but primarily in the relatively undetectable condition $\left(\mathrm{E} 2=3.11^{\circ}\right)$ - in the more detectable filtering condition $\left(\mathrm{E} 2=1.55^{\circ}\right)$ the trend was somewhat less clear, and of course it was absent in the undetectable filtering condition $\left(\mathrm{E} 2=6.22^{\circ}\right)$. This also suggests a 3-way interaction between filtering level, saccade length, and update delay, but again the statistical analyses did not support it. As noted above, the current study lacked a sufficiently large pool of data to effectively test these fine-grained hypotheses. Thus, although 12 subjects' data was sufficient to show clear effects of update delays on detection of blur, particularly in the rarely detectable blur condition $\left(\mathrm{E} 2=3.11^{\circ}\right)$, further research with a larger number of subjects may be necessary to clarify the possible 3-way interaction between saccade length, filtering level, and update delays on blur detection. Nevertheless, if the above analysis is correct, it suggests that detectible visual artifacts caused by long update delays would be most likely to occur in situations in which viewers tend to make large eye movements (e.g., with large displays, such as VR, having relatively large distances between objects). In such cases, if faster updates are not possible, a simple solution would be to increase the size of the region of highest resolution, or equivalently, to use a somewhat shallower image resolution drop-off function [for a more sophisticated version of this idea, see Komogortsev and Khan 2004]. This would reduce the detectability of image blur and delay-based motion transients, though at the cost of somewhat reducing the processing and bandwidth savings. 


\section{ACKNOWLEDGMENTS}

The authors wish to acknowledge George McConkie for helpful feedback and use of his laboratory, and Jian Yang, who developed the multi-resolutional image processing software, both of which made this research possible.

\section{REFERENCES}

BURR, D.C., MORRONE, M.C. and ROSS, J. 1994. Selective suppression of the magnocellular visual pathway during saccadic eye movements. Nature, 371 (6497). 511-513.

DRAPER, M.H., VIIRRE, E.S., FURNESS, T.A. and GAWRON, V.J. 2001. Effects of image scale and system time delay on simulator sickness within head-coupled virtual environments. Human Factors, 43 (1). 129-146.

DUCHOWSKI, A.T. and COLTEKIN, A. 2007. Foveated Gaze-contingent Displays for peripheral LOD management, 3D visualization, and stereo imaging. Transactions on Multimedia Computing, Communications, and Applications (this issue).

DUCHOWSKI, A.T., COURNIA, N. and MURPHY, H. 2004. Gaze-Contingent Displays: A Review. CyberPsychology \& Behavior, 7 (6). 621-634.

FRANK, L.H., CASALI, J.G. and WIERWILLE, W.W. 1988. Effects of visual display and motion system delays on operator performance and uneasiness in a driving simulator. Human Factors, 30 (2). 201-217.

GEISLER, W.S. and PERRY, J.S. 1998. A real-time foveated multi-resolution system for low-bandwidth video communication. Proceedings of the SPIE: The International Society for Optical Engineering, 3299. 294-305.

GEISLER, W.S. and PERRY, J.S. 1999. Variable-Resolution Displays for Visual Communication and Simulation. in The Society for Information Display, 1999, 420-423.

GERI, G.A. and ZEEVI, Y.Y. 1995. Visual Assessment of Variable-Resolution Imagery. Journal of the Optical Society of America A-Optics \& Image Science, 12 (10). 2367-2375.

GRUNWALD, A.J. and KOHN, S. 1994. Visual field information in low-altitude visual flight by line-of-sight slaved helmet-mounted displays. IEEE Transactions on Systems, Man and Cybernetics, 24 (1). 120-134.

HODGSON, T.L., MURRAY, P.M. and PLUMMER, A.R. 1993. Eye movements during "area of interest" viewing. in d'Ydewalle, G. and Rensbergen, J.V. eds. Perception and cognition: Advances in eye movement research, Elsevier Science, New York, 1993, 115-123.

KOMOGORTSEV, O. and KHAN, J., Predictive perceptual compression for real time video communication. in 12th annual ACM international conference on Multimedia, (New York, NY, USA, 2004), ACM Press, 220227.

KORTUM, P.T. and GEISLER, W.S., Search performance in natural scenes: The role of peripheral vision. in ARVO, (1996).

LOSCHKY, L.C. and MCCONKIE, G.W. 2002. Investigating spatial vision and dynamic attentional selection using a gaze-contingent multi-resolutional display. Journal of Experimental Psychology: Applied, 8 (2). 99117.

LOSCHKY, L.C. and MCCONKIE, G.W. 2000. User performance with gaze contingent multiresolutional displays. in Duchowski, A.T. ed. Proceedings of the Eye Tracking Research \& Applications Symposium 2000, ACM, Palm Beach, FL., 2000, 97-103.

LOSCHKY, L.C., MCCONKIE, G.W., YANG, J. and MILLER, M.E. 2005. The limits of visual resolution in natural scene viewing. Visual Cognition, 12 (6). 1057-1092.

LUEBKE, D., HALLEN, B., NEWFIELD, D. and WATSON, B. 2000. Perceptually Driven Simplification Using Gaze-Directed Rendering Technical Report CS-2000-04, University of Virginia, Department of Computer Science, Charlottesville, 2000.

MCCONKIE, G.W. and LOSCHKY, L.C. 2002. Perception onset time during fixations in free viewing. Behavioral Research Methods, Instruments, and Computers, 34 (4). 481-490.

OHSHIMA, T., YAMAMOTO, H. and TAMURA, H. 1996. Gaze-directed adaptive rendering for interacting with virtual space. in Virtual Reality Annual International Symposium, IEEE, 1996, 103-110.

PARKHURST, D., CULURCIELLO, E. and NEIBUR, E. 2000. Evaluating variable resolution displays with visual search: Task performance and eye movements. in Duchowski, A.T. ed. Proceedings of the Eye Tracking Research \& Applications Symposium 2000, ACM, Palm Beach, FL., 2000, 105-109.

PARKHURST, D.J. and NIEBUR, E. 2002. Variable resolution displays: a theoretical, practical, and behavioral evaluation. Human Factors, 44. 611-629.

REINGOLD, E.M. and LOSCHKY, L.C. 2002. Saliency of peripheral targets in gaze-contingent multiresolutional displays. Behavior Research Methods, Instruments and Computers, 34 (4). 491-499.

REINGOLD, E.M., LOSCHKY, L.C., MCCONKIE, G.W. and STAMPE, D.M. 2003. Gaze-contingent multiresolutional displays: An integrative review. Human Factors, 45 (2). 307-328. 
REINGOLD, E.M. and STAMPE, D.M. 2000. Saccadic inhibition and gaze contingent research paradigms. in Kennedy, A., Radach, R., Heller, D. and Pynte, J. eds. Reading as a perceptual process, Elsevier, Amsterdam, 2000, 119-145.

ROSS, J., MORRONE, M.C., GOLDBERG, M.E. and BURR, D.C. 2001. Changes in visual perception at the time of saccades. Trends in Neurosciences, 24 (2). 113-121.

SERE, B., MARENDAZ, C. and HERAULT, J. 2000. Nonhomogeneous resolution of images of natural scenes. Perception, 29 (12). 1403-1412.

SHIOIRI, S. 1993. Postsaccadic processing of the retinal image during picture scanning. Perception \& Psychophysics, 53 (3). 305-314.

SHIOIRI, S. and IKEDA, M. 1989. Useful resolution for picture perception as a function of eccentricity. Perception, 18. 347-361.

THOMAS, M. and GELTMACHER, H. 1993. Combat simulator display development. Information Display, 9. 23-26.

TURNER, J.A. 1984. Evaluation of an eye-slaved area-of-interest display for tactical combat simulation. in The 6th Interservice/Industry Training Equipment Conference and Exhibition, 1984, 75-86.

VAN DIEPEN, P.M.J. and WAMPERS, M. 1998. Scene exploration with Fourier-filtered peripheral information. Perception, 27 (10). 1141-1151.

VOLKMANN, F.C., RIGGS, L.A., WHITE, K.D. and MOORE, R.K. 1978. Contrast sensitivity during saccadic eye movements. Vision Research, 18 (9). 1978, 1193-1199.

WATSON, B.A., WALKER, N., HODGES, L.F. and WORDEN, A. 1997. Managing level of detail through peripheral degradation: Effects on search performance with a head-mounted display. ACM Transactions on Computer-Human Interaction, 4 (4). 323-346.

YANG, J., COIA, T. and MILLER, M. 2001. Subjective evaluation of retinal-dependent image degradations. in Proceedings of PICS 2001: Image Processing, Image Quality, Image Capture Systems Conference, The Society for Imaging Science and Technology, Springfield, VA, 2001, 142-147. 